\title{
SYMMETRIC MULTIPARAMETER PROBLEMS AND DEFICIENCY INDEX THEORY
}

\author{
by PATRICK J. BROWNE* and HAMLET ISAEV
}

(Received 10th March 1987)

\section{Introduction}

In this article we study the multiparameter generalization of standard deficiency index theory. A classical result in this area states that if $T$ is a symmetric operator in a Hilbert space then the dimension of the null space of $T^{*}-\lambda I, \lambda \in \mathbb{C}$, is constant for $\lambda$ belonging to the upper (or lower) half-plane and further, when these two constants are equal, $T$ admits a self-adjoint extension.

The multiparameter problem to be discussed can be posed as follows. Let $H_{1}, \ldots, H_{k}$ be Hilbert spaces and consider operators

(i) $T_{r}: D\left(T_{r}\right) \subset H_{r} \rightarrow H_{r}, \overline{D\left(T_{r}\right)}=H_{r}, T_{r} \subset T_{r}^{*}, T_{r}$ closed,

(ii) $V_{r s}: H_{r} \rightarrow H_{r}, V_{r s}=V_{r s}^{*}, V_{r s}$ bounded, $1 \leqq r, s \leqq k$.

It is customary to assume some definiteness condition on the array of operators $\left[V_{r s}\right]$ and here we shall impose what is known as uniform right definiteness (URD). This can be described as follows. Let

$$
V_{r s}^{+}=I \otimes \cdots \otimes V_{r s} \otimes \cdots \otimes I: H \rightarrow H \text { where } H=\bigotimes_{r=1}^{k} H_{r} .
$$

The operator $\Delta_{0}$ is then defined as

$$
\Delta_{0}=\operatorname{det}\left[V_{r s}^{+}\right]
$$

where the determinant is expanded formally. This construction is standard in multiparameter theory-see the survey paper [3], the monograph [9] or the lecture notes [7] for compendia of recent results in the area. Our definiteness condition, then, is:

URD: $\Delta_{0} \geqq c l$ on $H$ for some $c>0$.

For $\lambda=\left(\lambda_{1}, \ldots, \lambda_{k}\right) \in \mathbb{C}^{k}$, we put

$$
W_{r}(\lambda)=T_{r}-\sum_{s=1}^{k} \lambda_{s} V_{r s}: D\left(T_{r}\right) \subset H_{r} \rightarrow H_{r}, \quad 1 \leqq r \leqq k .
$$

* Research supported in part by the N.S.E.R.C. of Canada. 
Our first task is to investigate

$$
W_{r}(\bar{\lambda})^{*}=T_{r}^{*}-\sum_{s=1}^{k} \lambda_{s} V_{r s}: D\left(T_{r}^{*}\right) \subset H_{r} \rightarrow H_{r}, \quad 1 \leqq r \leqq k,
$$

and to find regions in $\mathbb{C}^{k}$ where the dimensions of the null spaces of these operators are constant. We evaluate these constants in terms of the deficiency indices of $T_{r}$. These results make up Section 2.

Secondly we shall consider the operators $\Phi_{1}, \ldots, \Phi_{k}$ defined on the algebraic tensor product $\bigotimes_{r=1}^{k} D\left(T_{r}^{*}\right)$ by

$$
\Phi_{1}=\operatorname{det}\left|\begin{array}{ccc}
T_{1}^{*} & V_{12} \ldots & V_{1 k} \\
\vdots & & \\
T_{k}^{*} & V_{k 2} \ldots & V_{k k}
\end{array}\right|, \ldots, \Phi_{k}=\operatorname{det}\left|\begin{array}{ccc}
V_{11} & \ldots & T_{1}^{*} \\
\vdots & & \\
V_{k 1} & \ldots & T_{k}^{*}
\end{array}\right|,
$$

where again the determinants are to be expanded formally. We put $\Omega_{r}=\Delta_{0}^{-1} \Phi_{r}, 1 \leqq r \leqq k$, and we draw a connection between solutions of $\left(\Omega_{r}-\lambda_{r} I\right) x=0$ and of $W_{r}(\bar{\lambda})^{*} x_{r}=0$, $1 \leqq r \leqq k$. Specific contributions to this multiparameter problem for systems of ordinary differential equations can be found in [5], [10].

We close with some open questions in the area.

\section{Deficiency indices}

We use the notation of the introduction. For $1 \leqq r \leqq k$, we define the sets

$$
\begin{aligned}
& M_{r}^{+}=\left\{\lambda \in \mathbb{C}^{k} \mid \sum_{s=1}^{k}\left(\operatorname{Im} \lambda_{s}\right) V_{r s} \gg 0 \text { on } H_{r}\right\}, \\
& M_{r}^{-}=\left\{\lambda \in \mathbb{C}^{k} \mid \sum_{s=1}^{k}\left(\operatorname{Im} \lambda_{s}\right) V_{r s} \ll 0 \text { on } H_{r}\right\} .
\end{aligned}
$$

Here, for an operator $A$ on a Hilbert space, $A \gg 0$ means $A \geqq \alpha I$ for some $\alpha>0$. Our definiteness condition URD implies that $M_{r}^{+} \neq \Phi, M_{r}^{-} \neq \phi, 1 \leqq r \leqq k-$ (see [2, Theorem 2]). The following properties are easy to establish.

Proposition 2.1. For each $1 \leqq r \leqq k$,

(i) $M_{r}^{+}, M_{r}^{-}$are open and convex,

(ii) $M_{r}^{-}=-M_{r}^{+}=\left(M_{r}^{+}\right)^{*}\left(=\left\{\lambda \mid \lambda \in M_{r}^{+}\right)\right.$.

Our first result is:

Theorem 2.2. We have dim $\operatorname{ker}\left[W_{r}(\lambda)^{*}\right]$ is constant for $\lambda \in M_{r}^{+}$and for $\lambda \in M_{r}^{-}$.

Proof. Let $\lambda^{0} \in M_{r}^{+}$and consider $x_{r} \in D\left(T_{r}\right)$. Then 


$$
\begin{aligned}
& \left(W_{r}\left(\bar{\lambda}^{0}\right) x_{r}, x_{r}\right)=\left(T_{r} x_{r}, x_{r}\right)-\left(\sum_{s=1}^{k} \lambda_{s}^{0} V_{r s} x_{r}, x_{r}\right), \\
& \left(x_{r}, W_{r}\left(\lambda^{0}\right) x_{r}\right)=\left(T_{r} x_{r}, x_{r}\right)-\left(\sum_{s=1}^{k} \lambda_{s}^{0} V_{r s} x_{r}, x_{r}\right) .
\end{aligned}
$$

Thus we have

$$
\begin{aligned}
\left\|W_{r}\left(\bar{\lambda}^{0}\right) x_{r}\right\|\left\|x_{r}\right\| & \geqq\left|\left(W_{r}\left(\lambda^{0}\right) x_{r}, x_{r}\right)\right| \\
& \geqq\left|\operatorname{Im}\left(W_{r}\left(\bar{\lambda}^{0}\right) x_{r}, x_{r}\right)\right| \\
& =\left|\left(\sum_{s=1}^{k}\left(\operatorname{Im} \lambda_{s}^{0}\right) V_{r s} x_{r}, x_{r}\right)\right| \\
& \geqq \alpha_{r}\left\|x_{r}\right\|^{2}, \text { for some } \alpha_{r}>0 .
\end{aligned}
$$

Hence

$$
\left\|W_{r}\left(\bar{\lambda}^{0}\right) x_{r}\right\| \geqq \alpha_{r}\left\|x_{r}\right\| \quad \text { for all } x_{r} \in D\left(T_{r}\right) \text {. }
$$

Now if $\lambda \in M_{r}^{+}$, then

$$
W_{r}(\bar{\lambda})=W_{r}\left(\bar{\lambda}^{0}\right)-\sum_{s=1}^{k}\left(\overline{\lambda_{s}}-\overline{\lambda_{s}^{0}}\right) V_{r s}
$$

and if $\lambda$ is such that $\left|\lambda_{s}-\lambda_{s}^{0}\right|<\alpha_{r} / \sum_{s=1}^{k} \| V_{r s}||$, then

$$
\left\|\sum_{s=1}^{k}\left(\bar{\lambda}_{s}-\bar{\lambda}_{s}^{0}\right) V_{r s}\right\|<\alpha_{r}
$$

The following lemma from perturbation theory is an easy consequence of [4, Corollary V.1.3, p. 111].

Lemma 2.3. Let $A$ be a closed operator and B a bounded operator in a Hilbert space, satisfying

$$
\|B\|<\alpha,\|A x\| \geqq \alpha\|x\|, x \in D(A), \text { for some } \alpha>0 .
$$

Then

$$
\operatorname{dim} \operatorname{ker}\left(A^{*}\right)=\operatorname{dim} \operatorname{ker}\left(A^{*}+B^{*}\right) .
$$

Returning to the proof of our theorem, we use the lemma with $A=W_{r}\left(\bar{\lambda}^{0}\right)$ and $B=-\sum_{s=1}^{k}\left(\bar{\lambda}_{s}-\bar{\lambda}_{s}^{0}\right) V_{r s}$. The result is now immediate for the lemma shows $\operatorname{dim} \operatorname{ker}\left[W_{r}(\bar{\lambda})^{*}\right]$ 
to be a local constant in $M_{r}^{+}$and the topological properties of $M_{r}^{+}$show this dimension to be a global constant in $M_{r}^{+}$. The discussion for $M_{r}^{-}$follows similar lines and so the theorem is established.

Our next task is to evaluate the two constants produced in the theorem above. We denote by $n_{r}^{+}, n_{r}^{-}$the deficiency indices of $T_{r}$, i.e.

$$
n_{r}^{+}=\operatorname{dim} \operatorname{ker}\left[T_{r}^{*}-i \Gamma\right], n_{r}^{-}=\operatorname{dim} \operatorname{ker}\left[T_{r}^{*}+i I\right] .
$$

Theorem 2.4. If $\lambda \in M_{r}^{+}\left(\right.$respectively, $\left.M_{r}^{-}\right)$then

$$
\operatorname{dim} \operatorname{ker}\left[W_{r}(\bar{\lambda})^{*}\right]=n_{r}^{+}\left(\text {respectively, } n_{r}^{-}\right) .
$$

Proof. We consider the $(k+2)$ operators in $H_{r}$

$$
T_{r} V_{r 1} \ldots V_{r k} I
$$

and define

$$
\hat{M}_{r}^{+}=\left\{\lambda \in \mathbb{C}^{k+1} \mid \sum_{s=1}^{k} \operatorname{Im} \lambda_{s} V_{r s}+\left(\operatorname{Im} \lambda_{k+1}\right) I \gg 0\right\} .
$$

We note that $\lambda \in M_{r}^{+}$implies $(\lambda, 0) \in \hat{M}_{r}^{+}$and also $(0, \ldots, 0, i) \in \hat{M}_{r}^{+}$. Thus we take $\lambda \in M_{r}^{+}$ and apply Theorem 2.1 to this larger system of operators to obtain

$$
\operatorname{dim} \operatorname{ker}\left[W_{r}(\bar{\lambda})^{*}\right]=\operatorname{dim} \operatorname{ker}\left[T_{r}^{*}-i I\right]=n_{r}^{+} .
$$

The argument for $\lambda \in M_{r}^{-}$is identical.

We may now appeal to the well known properties of the deficiency indices for symmetric operators-[1, Chapter 8$]$ is a suitable reference, to claim the following

Corollary 2.5. (i) $T_{r}$ has a self-adjoint extension if, and only if, there is a point $\lambda \in M_{r}^{+}$ such that

$$
\operatorname{dim} \operatorname{ker}\left[W_{r}(\bar{\lambda})^{*}\right]=\operatorname{dim} \operatorname{ker}\left[W_{r}(\lambda)^{*}\right] .
$$

(ii) If $T_{r}$ is semi-bounded and $\lambda \in M_{r}^{+}$then (2.1) holds.

(iii) If $Q_{r}$ is bounded and symmetric on $H_{r}$ then for $\lambda \in M_{r}^{+}$,

$$
\operatorname{dim} \operatorname{ker}\left[W_{r}(\bar{\lambda})^{*}+Q_{r}\right]=\operatorname{dim} \operatorname{ker}\left[W_{r}(\bar{\lambda})\right]=n_{r}^{+} .
$$

(iv) Suppose $T_{r}$ is bounded below and $\lambda=\sigma+i t \in M_{r}^{+}, \sigma, t \in \mathbb{R}^{k}$. Then there is a real 
number $\theta_{0}>0$ so that if $\theta>\theta_{0}$ a self-adjoint extension $T_{r}(\theta)$ of $T_{r}$ can be found so that

$$
\left(\tilde{T}_{r}(\theta)+\sum_{s=1}^{k} \theta t_{s} V_{r s}\right) x_{r}=0 \quad \text { for some } x_{r} \neq 0 .
$$

Proof. Claims (i), (ii), (iii) are easy. For (iv) we note that $\sum_{s=1}^{k} t_{s} V_{r s} \gg 0$ so we select $\theta_{0}$ large enough to satisfy

$$
T_{r}+\sum_{s=1}^{k} \theta_{0} t_{s} V_{r s} \gg 0
$$

The result now follows from [1, Theorem 3, p. 365].

\section{Decoupling the spectral parameters}

In this section we study the process of decoupling the spectral parameters $\lambda_{1}, \ldots, \lambda_{k}$ in a system of simultaneous equations $W_{r}(\lambda)^{*} x_{r}=0,1 \leqq r \leqq k$. This is a commonly used idea in (self-adjoint) multiparameter spectral theory and, from some points of view, forms the basis for it. We begin with some preliminaries.

Note that the operators $I \bigotimes_{a} \cdots \bigotimes_{a} T_{r} \bigotimes_{a} \cdots \bigotimes_{a} I$ and $I \bigotimes_{a} \cdots \bigotimes_{a} T_{r}^{*} \bigotimes_{a} \cdots \bigotimes_{a} I$ are closeable since they are both restrictions of the closed operator $\left(I \otimes_{a} \cdots \bigotimes_{a} T_{r} \bigotimes_{a} \cdots \bigotimes_{a} I\right)^{*}$. We shall use $T_{r}^{+}$and $T_{r}^{*+}$ to denote these closures. The operators $W_{r}(\lambda)^{+}$and $W_{r}(\lambda)^{*+}$ are defined in like fashion.

Lemma 3.1. Let $\lambda \in \mathbb{C}^{k}$. Then

$$
\bigotimes_{r=1}^{k} \operatorname{ker}\left[W_{r}(\bar{\lambda})^{*}\right]=\bigcap_{r=1}^{k} \operatorname{ker}\left[W_{r}(\bar{\lambda})^{*+}\right]
$$

Proof. We should point out that the tensor product on the left hand side above is the Hilbert tensor product, i.e. it is the closure in $H$ of the algebraic tensor product of the closed subspaces $\operatorname{ker}\left[W_{r}(\lambda)^{*}\right]$ and, as such, it is a closed subspace of $H$. The right hand side is also a closed subspace of $H$ as it is the intersection of the kernels of the closed operators $W_{r}(\bar{\lambda})^{*+}$.

It is easy to see

$$
\bigotimes_{r=1^{a}}^{k} \operatorname{ker}\left[W_{r}(\bar{\lambda})^{*}\right] \subset \bigcap_{r=1}^{k} \operatorname{ker}\left[W_{r}(\bar{\lambda})^{*+}\right]
$$

and so we take closures to obtain

$$
\bigotimes_{r=1}^{k} \operatorname{ker}\left[W_{r}(\lambda)^{*}\right] \subset \bigcap_{r=1}^{k} \operatorname{ker}\left[W_{r}(\lambda)^{*+}\right] .
$$

Now let $\operatorname{ker}\left[W_{r}(\bar{\lambda})^{*}\right]$ have an orthonormal basis $\left\{e_{r}^{1}, e_{r}^{2}, \ldots\right\}$ and its orthocomplement 
have an orthonormal basis $\left\{f_{r}^{1}, f_{r}^{2}, \ldots\right\}$. We show that any tensor of the form $q=$ $x_{1} \otimes \cdots \otimes f_{r}^{j} \otimes \cdots \otimes x_{k}$ belongs to $\left(\operatorname{ker}\left[W_{r}\left(\lambda^{*+}\right]\right)^{\perp}\right.$. To see this, first note that $f_{r}^{j} \in\left(\operatorname{ker}\left[W_{r}(\bar{\lambda})^{*}\right]\right)^{\perp}=\bar{R}\left[W_{r}(\bar{\lambda})\right]$, and so $f_{r}^{j}=\lim _{n \rightarrow \infty} W_{r}(\bar{\lambda}) y_{r}^{n}, y_{r}^{n} \in D\left(T_{r}\right)$. From our discussion and definitions at the start of this section it also follows that

$$
W_{r}(\lambda)^{*+} \subset\left(W_{r}(\lambda)^{+}\right)^{*}
$$

Hence if $z \in \operatorname{ker}\left[W_{r}(\bar{\lambda})^{*+}\right]$, we have

$$
\begin{aligned}
(q, z) & =\lim _{n \rightarrow \infty}\left(x_{1} \otimes \cdots \otimes W_{r}(\bar{\lambda}) y_{r}^{n} \otimes \cdots \otimes x_{k}, z\right) \\
& =\lim _{n \rightarrow \infty}\left(x_{1} \otimes \cdots \otimes y_{r}^{n} \otimes \cdots \otimes x_{k}, W_{r}(\bar{\lambda})^{*+} z\right) \\
& =0,
\end{aligned}
$$

establishing our claim.

Vectors of the form $x_{1} \otimes \cdots \otimes x_{k}$ where $x_{r} \in\left\{e_{r}^{1}, e_{r}^{2}, \ldots, f_{r}^{1}, f_{r}^{2}, \ldots\right\}$ form an orthonormal basis for $H$. Those of the type in which $x_{r} \in\left\{e_{r}^{1}, e_{r}^{2}, \ldots\right\}, 1 \leqq r \leqq k$, lie within $\bigcap_{r=1}^{k} \operatorname{ker}\left[W_{r}(\bar{\lambda})^{*+}\right]$ by our opening remarks and the remainder lie within $\left(\bigcap_{r=1}^{k} \operatorname{ker}\left[W_{r}(\bar{\lambda})^{*+}\right]\right)^{\perp}$ by the argument above. From this observation, the lemma follows immediately. A more general version of this result can be found in [6].

We now proceed with the decoupling process by first noting that with $\Phi_{1}, \ldots, \Phi_{k}$ as defined in the introduction and $\Omega_{s}=\Delta_{0}^{-1} \Phi_{s}, 1 \leqq s \leqq k$, we have

$$
T_{r}^{*+}-\sum_{s=1}^{k} V_{r s}^{+} \Omega_{s}=0 \quad \text { on } \bigotimes_{r=1^{a}}^{k} D\left(T_{r}^{*}\right) \text {. }
$$

This follows readily from [5, Theorem 2] which states that when $x \in \bigotimes_{r=1 a}^{k} D\left(T_{r}^{*}\right)$, the equations

$$
T_{r}^{*+} x-\sum_{s=1}^{k} V_{r s}^{+} g^{s}=0, \quad 1 \leqq r \leqq k,
$$

can be solved uniquely for $g^{1}, \ldots, g^{k}$ with $g^{k}=\Omega_{s}$. In fact we can use (3.1) to extend the domain of definition of each $\Omega_{s}$ from $\otimes_{r=1 a}^{k} D\left(T_{r}^{*}\right)$ to $\bigcap_{r=1}^{k} D\left(T_{r}^{*+}\right)$. We continue to write $\Omega_{s}$ for this extension.

Theorem 3.2. Let $\lambda \in \mathbb{C}^{k}$. Then

$$
\bigotimes_{r=1}^{k} \operatorname{ker}\left[W_{r}(\bar{\lambda})^{*}\right]=\bigcap_{r=1}^{k} \operatorname{ker}\left[W_{r}(\bar{\lambda})^{*+}\right]=\bigcap_{r=1}^{k} \operatorname{ker}\left[\Omega_{r}-\lambda_{r} I\right]
$$

Proof. In view of the previous lemma, only the second equality requires discussion. If $x \in \bigcap_{r=1}^{k} D\left(T_{r}^{*+}\right)$ and 


$$
\left(T_{r}^{*+}-\sum_{s=1}^{k} \lambda_{s} V_{r s}^{+}\right) x=0,1 \leqq r \leqq k
$$

then it follows that

$$
\sum_{s=1}^{k} V_{r s}^{+}\left(\Omega_{s}-\lambda_{s} I\right) x=0,1 \leqq r \leqq k
$$

We again use [5, Theorem 2] to deduce that $x \in \bigcap_{r=1}^{k} \operatorname{ker}\left[\Omega_{s}-\lambda_{s} I\right]$. The reverse argument is similar.

Definition 3.3. For $\lambda \in \mathbb{C}^{k}$ we define the deficiency index for the system $\left[T, V_{r s}\right]_{r, s=1}^{k}$ at $\lambda$ to be

$$
\begin{aligned}
N(\lambda) & =\prod_{r=1}^{k} \operatorname{dim} \operatorname{ker}\left[W_{r}(\bar{\lambda})^{*}\right] \\
& =\operatorname{dim} \bigcap_{r=1}^{k} \operatorname{ker}\left[\Omega_{r}-\lambda_{r} I\right] .
\end{aligned}
$$

For $\varepsilon=\left(\varepsilon_{1}, \ldots, e_{k}\right), \varepsilon_{r}= \pm 1,1 \leqq r \leqq k$, we put

$$
M_{e}=\left\{\lambda \in \mathbb{C}^{k} \mid \varepsilon_{r} \sum_{s=1}^{k}\left(\operatorname{Im} \lambda_{s}\right) V_{r s} \gg 0,1 \leqq r \leqq k\right\} .
$$

From [2, Theorem 2] we know that $M_{\varepsilon} \neq \Phi$. In fact

$$
M_{e}=\bigcap_{r=1}^{k} M_{r}^{\varepsilon_{r}}
$$

Corollary 3.4. For each $\varepsilon, N(\lambda)$ is constant in $M_{\varepsilon}$. In fact if $\lambda \in M_{\varepsilon}$,

$$
N(\lambda)=\prod_{r=1}^{k} n_{r}^{e_{r}}
$$

Proof. This is an immediate consequence of Theorem 2.4.

\section{Open questions}

When the operators $T_{r}, 1 \leqq r \leqq k$, are self-adjoint it is known from standard multiparameter theory that $\Omega_{1}, \ldots, \Omega_{k}$ are self-adjoint in $H$ equipped with the inner product $[x, y]=\left(\Delta_{0} x, y\right)$ and are pairwise commuting in the sense that their spectral measures commute. If each $T_{r}$ is symmetric and has equal deficiency indices then it follows that $\Omega_{1}, \ldots, \Omega_{k}$ have restrictions which are $[\cdot, \cdot]$-self-adjoint and pairwise commutative. Is it possible for $\Omega_{1}, \ldots, \Omega_{k}$ to have such restrictions when the operators $T_{r}$ do not have self- 
adjoint extension? If $\Gamma_{1}, \ldots, \Gamma_{k}$ are such restrictions of $\Omega_{1}, \ldots, \Omega_{k}$, is it possible to characterize the corresponding extensions to $T_{1}, \ldots, T_{k}$ ?

Acknowledgement. One of us, (PJB), wishes to thank the Institute for Mathematics and Mechanics, Academy of Sciences of Azerbaijan S.S.R., Baku, for its warm hospitality during September, 1986, when the authors prepared these results.

\section{REFERENCES}

1. N. I. Akhiezer and I. M. Glazman, Theory of Linear Operators in Hilbert Space, Vol. II (Pitman, London, 1981).

2. P. Binding and P. J. Browne, Positivity results for determinantal operators, Proc. Roy. Soc. Edinburgh 81A (1978), 267-271.

3. P. J. Browne, Multiparameter problems: the last decade, Proceedings 1982 Dundee Conference on Ordinary and Partial Differential Equations (Springer-Verlag Lecture Notes in Mathematics, Vol. 964), 95-109.

4. S. GoldBerG, Unbounded Linear Operators (McGraw-Hill, New York, 1966).

5. H. A. IsAEv, On the theory of deficiency indices for multiparameter differential operators of Sturm-Liouville type, Soviet Math. Dokl. 24 (1981), 580-583.

6. H. A. IsAev, Introduction to general multiparameter spectral theory (Russian), Spectral Theory of Operators, Vol. 3 ("Elm", Baku, 1980), 142-201.

7. H. A. IsAev, Lectures on multiparameter spectral theory (Department of Mathematics and Statistics, University of Calgary, 1985).

8. A. Källstróm and B. D. Sleeman, Solvability of a linear operator system, J. Math. Anal. Appl. 55 (1976), 785-793.

9. B. D. Sleeman, Multiparameter Spectral Theory in Hilbert Space (Pitman, London, 1978).

10. B. D. Sleeman, Singular linear differential operators with many parameters, Proc. Roy. Soc. Edinburgh 71(A) (1973), 199-232.

Department of Mathematics and Statistics

UNIVERSITY OF CALGARY

Calgary, Alberta

INSTITUTE for Mathematics and Mechanics

CANADA T2N 1N4 ACADEMy of Science of Azerbaijan S.S.R.

BAKU

U.S.S.R. 